Remarkable rigidity of the single $\alpha$-helical domain of myosin-VI revealed by NMR spectroscopy

C. Ashley Barnes, ${ }^{a}$ Yang Shen, ${ }^{a}$ Jinfa Ying, ${ }^{a}$ Yasuharu Takagi, ${ }^{b}$ Dennis A. Torchia, ${ }^{a}$ James R. Sellers ${ }^{\mathrm{b}}$ and $\mathrm{Ad} \mathrm{Bax}{ }^{\mathrm{a},{ }^{*}}$

${ }^{a}$ Laboratory of Chemical Physics, NIDDK,

${ }^{\mathrm{b}}$ Laboratory of Molecular Physiology, NHLBI

National Institutes of Health, Bethesda, MD, 20892-0520, USA 


\section{Hydrodynamic modeling}

Assuming a protein density of $1.4 \mathrm{~g} / \mathrm{cm}^{3}$, and a mass of $7.96 \mathrm{kD}$ for the center 60 residues of the MT domain, we modeled this section of the domain as a cylinder with a volume of $10,000 \AA^{3}$. With an unhydrated length of $60 \times 1.5 \AA$, this yields a radius of $6 \AA$ prior to making the "bare atom correction" of $2 \AA,{ }^{1}$ effectively accounting for the presence of protein hydrogen atoms at the molecular surface, and an additional $1-\AA \AA$ correction to account for the hydration layer. ${ }^{1}$ Adding these corrections then results in a model cylinder of length $l_{\text {cyl }}=96 \AA$ and a radius $r_{\text {cyl }}=9 \AA$. Rotational diffusion of an axially symmetric rotor such as cylinder is characterized by two diffusion constants $\mathrm{D} / /$ and $\mathrm{D} \perp$, describing rotation about the long (symmetry) and short axes of the cylinder, respectively. The correlation functions needed to calculate $\mathrm{R}_{1}, \mathrm{R}_{2}$ and the NOE depend upon (a) the angle made by the internuclear $\mathrm{N}-\mathrm{H}$ vector and the long axis of the helix, $14^{\circ}$, for an ideal $\alpha$-helix, and (b) three correlation times $\tau_{\mathrm{A}}, \tau_{\mathrm{B}}$ and $\tau_{\mathrm{C}}$ given by $\tau_{\mathrm{A}}=1 / 6 \mathrm{D} \perp ; \tau_{\mathrm{B}}=1 /(\mathrm{D} / /+5 \mathrm{D} \perp)$; $\tau_{\mathrm{C}}=1 /(4 \mathrm{D} / /+2 \mathrm{D} \perp)$. Since $1_{\text {cyl }}>>\mathrm{r}_{\text {cyl }}, \mathrm{D} / />\mathrm{D} \perp$ and hence $\tau_{\mathrm{A}}>\tau_{\mathrm{B}}>\tau_{\mathrm{C}}$. Herein we employ expressions of Garcia de la Torre and Bloomfield ${ }^{2-3}$ which yield $\mathrm{D} / / / \mathrm{D} \perp=7.6$ and $\tau_{\mathrm{m}}=$ $1 /(2 \mathrm{D} / /+4 \mathrm{D} \perp)=10.3 \mathrm{~ns}$.

\section{${ }^{1} \mathbf{H}-{ }^{1} \mathbf{H}$ NOE simulations}

The signal intensities of NOESY cross-peaks of protons $i$ and $j$ are proportional to proton-proton cross-relaxation rates, $\sigma_{\mathrm{ij}}$. The $\sigma_{\mathrm{ij}}$ are functions of (a) the overall correlation times of the helix, (b) the internuclear $\mathrm{H}_{\mathrm{i}}-\mathrm{H}_{\mathrm{j}}$ distance $\left(2.8 \AA\right.$ for $\left.\mathrm{H}_{\mathrm{i}}-\mathrm{H}_{\mathrm{i} \pm 1}\right)$, and (c) the angle made by the $\mathrm{H}_{\mathrm{i}}-\mathrm{H}_{\mathrm{j}}$ internuclear vectors and the long axis of the helix. In addition, an order parameter $\boldsymbol{S}_{\mathrm{HH}}{ }^{2}$ that accounts for internal motion of the $\mathrm{H}_{\mathrm{i}}-\mathrm{H}_{\mathrm{j}}$ internuclear vector scales $\sigma_{\mathrm{ij}}$. The NOESY spectrum was recorded using a large mixing time $(0.25 \mathrm{~s})$ in order to ensure adequate signal-to-noise of the cross-peak intensities. Spin-diffusion effects were accounted for in the calculations by including cross-relaxation involving seven spins in the simulations of the cross-peak intensities (Figure S6A). The calculations also accounted for the fact that each amide site was $8 \%$ deuterated. Rate matrices and populations were calculated for the fully protonated state and the six states in which a single deuteron was present. The small population of remaining states (ca. 8\%) containing more than one deuteron, was divided by six and added to the population of each of the six single-deuteron states. The values of $\mathrm{I}_{ \pm \pm 1} / \mathrm{I}_{\mathrm{d}}$ so calculated are about $10 \%$ smaller than those calculated assuming $100 \%$ protonation. Cross-relaxation rates are calculated for an idealized a-helix with an axially symmetric diffusion tensor, with its unique axis parallel to the helix axis, an interproton $\mathrm{H}^{\mathrm{N}-} \mathrm{H}^{\mathrm{N}}$ distance of $2.8 \AA,{ }^{4} \tau_{\mathrm{m}}=10.3 \mathrm{~ns}$ and $\rho=7.6$. For a mixing time of 0.25 , the calculation yields a value of $\mathrm{I}_{ \pm 1} / \mathrm{I}_{\mathrm{d}}=0.33$, somewhat larger than the average measured value of $\mathrm{I}_{ \pm \pm 1} / \mathrm{I}_{\mathrm{d}}$, ca. 0.28 , for residues near the center of the helix. (Figure S6B). The observation that the calculated value of $\mathrm{I}_{\dot{1} \pm 1} / \mathrm{I}_{\mathrm{d}}$ exceeds the measured value, is in part due to the implicit assumption in the calculation that $S_{\mathrm{HH}^{2}}{ }^{2}=1$. Indeed, the observed decrease in $\mathrm{I}_{\mathrm{i} \pm 1} / \mathrm{I}_{\mathrm{d}}$ as residue positions recede from the center of the helix, following the behavior of ${ }^{15} \mathrm{~N} R_{2}$ (Figure 6C, main text). 


\section{Structure calculations}

Structural models for SAH were obtained using the XPLOR-NIH software package ${ }^{5}$ via a standard Cartesian molecular dynamics simulated annealing refinement protocol, starting from the coordinates of an idealized $\alpha$-helical structure with backbone torsion angles of $\phi=-62.5^{\circ}, \psi=$ $-42.5^{\circ}, \omega=180^{\circ}$. The protocol included 400,000 steps of $2 \mathrm{fs}$ each, with the temperature linearly ramped down from 3000 to $25 \mathrm{~K}$, followed by a Powell energy minimization. Fitted experimental restraints included the newly measured ${ }^{1} \mathrm{D}_{\mathrm{NH}},{ }^{1} \mathrm{D}_{\mathrm{C} N \mathrm{~N}},{ }^{2} \mathrm{D}_{\mathrm{C}^{\prime} \mathrm{H}} \mathrm{RDCs}$ from the Pf1 medium (with 100 $\mathrm{mM} \mathrm{NaCl}$ ) and ${ }^{1} \mathrm{D}_{\mathrm{HN}} \mathrm{RDCs}$ measured in Pf1 with no $\mathrm{NaCl}$, scaled by the inverse or the ${ }^{15} \mathrm{~N} \mathrm{R}_{2}$ to account for differential dynamics (see main text). Empirical force fields included quadratic bond, angle, and improper terms with force constants of $5000 \mathrm{kcal} . \AA^{-2} \cdot \mathrm{mol}, 500 \mathrm{kcal} \cdot \mathrm{rad}^{-2} \cdot \mathrm{mol}^{-1}$ and 500 kcal.rad ${ }^{-2} . \mathrm{mol}$, respectively, as well as a quartic repulsive-only non-bonded potential with a force constant of $4 \mathrm{kcal} . \AA^{-2} \cdot \mathrm{mol}^{-1}$. In addition, backbone/backbone hydrogen bonding geometries were restrained via a potential of mean force (HBDB term in XPLOR-NIH). ${ }^{6}$ Fixed magnitude alignment tensors were used during the structural calculations, with the axial and rhombic components of each alignment tensor determined by SVD fitting of the $\mathrm{R}_{2}$-scaled RDCs, of each alignment condition, to an idealized $\alpha$-helix. Force constants for different types of RDCs in two different alignment media were obtained from a combination of force constants $(0.4$ kcal. $\mathrm{Hz}^{-2} \cdot \mathrm{mol}^{-1}$ for ${ }^{1} D_{\mathrm{NH}} ; 0.1 \mathrm{kcal} \cdot \mathrm{Hz}^{-2} \cdot \mathrm{mol}^{-1}$ for ${ }^{1} D^{\prime} \mathrm{C}^{\prime} \mathrm{N} ; 0.2 \mathrm{kcal} \cdot \mathrm{Hz}^{-2} \cdot \mathrm{mol}^{-1}$ for ${ }^{2} D^{\prime} \mathrm{C}^{\prime} \mathrm{H}$, where ${ }^{1} D^{\prime}{ }_{C^{\prime} \mathrm{N}}=8.27 \times{ }^{1} D_{C^{\prime} \mathrm{N}}$ and ${ }^{2} D^{\prime} \mathrm{C}^{\prime} \mathrm{H}=3.10 \times{ }^{2} D_{\mathrm{C}^{\prime} \mathrm{H}}$, with ${ }^{1} D^{\prime} \mathrm{C}^{\prime} \mathrm{N}$ and ${ }^{2} D^{\prime} \mathrm{C}^{\prime} \mathrm{H}$ being the values normalized ${ }^{7}$ to the ${ }^{1} D_{\mathrm{NH}}$ couplings) that yielded best cross validation performance according to a grid searching procedure. The ${ }^{1} D_{\mathrm{NH}} \mathrm{RDC}$ force constant multipliers (and thereby the multipliers for the other types of RDCs) were ramped up with a constant multiplicative factor throughout the protocol from 0.05 to 2.5 ; i.e., at $25 \mathrm{~K}$, the ${ }^{1} D_{\mathrm{NH}}$ force constant was ramped up to $1 \mathrm{kcal} . \mathrm{Hz}^{-2} \cdot \mathrm{mol}^{-1}$.

The ten lowest energy structures obtained from the above refinement protocol were then used as starting conformers for modeling the side-chain conformations in implicit solvent using empirical energy terms of the XPLOR-NIH software. In addition to the energy terms used in the above backbone refinement protocol, two empirical terms, torsionDBPot and eefxPot, were used to model the side-chain conformations. A total of 50 structures was generated for each starting model, and the lowest energy structure was retained for each of the ten starting conformers and then deposited in the PDB (entry 6OBI).

\section{RDCs versus persistence length}

We derive the scaling of the averaged second order Legendre polynomial for a worm-like chain, $<P_{2}\left(\cos \left(\beta_{n}\right)\right)>$, as a function of position $n$ from the origin (tagging site), fully analogous to the derivation by Landau and Lifshitz ${ }^{8}$ of $\left\langle\cos \left(\beta_{n}\right)>=\exp \left(-n / L_{P}\right)\right.$. Following Landau and Lifshitz, the angles between the tangents, $t_{a}, t_{b}$, and $t_{c}$ at three points $a, b$, and $c$ on the chain are related by

$\cos \theta_{a c}=\cos \theta_{a b} \cos \theta_{b c}-\sin \theta_{a b} \sin \theta_{b c} \cos \phi_{a b, b c}$

where $\phi_{a b, b c}$ is the angle between the planes $\left(t_{a}, t_{b}\right)$ and $\left(t_{b}, t_{c}\right)$. Bearing in mind that in a worm-like chain the fluctuations of curvature for sections $a b$ and $b c$ for a given direction of $t_{b}$ are statistically independent, i.e., $<\cos \phi_{a b, b c}>=0$, averaging over a large distribution of worm like chains, all coaligned at the origin, yields 
$<\cos \theta_{a c}>=<\cos \theta_{a b}><\cos \theta_{b c}>$

Eq. A2 shows that the mean value of $q_{n}$ at position $n$ is a multiplicative function of the number of intervening segments, $n$, relative to the origin of the chain, i.e., must be an exponentially decreasing function $\exp \left(-n / L_{P}\right)$, with $L_{P}$ being the persistence length.

Below, we first show that analogous to eq A2, $<P_{2}\left(\cos \theta_{a c}\right)>=<P_{2}\left(\cos \theta_{a b}\right)><P_{2}\left(\cos \theta_{b c}\right)>$, i.e., that $<P_{2}\left(\cos \theta_{n}\right)>$ also is an exponentially decaying function:

$<P_{2}\left(\cos \theta_{a c}\right)>=<3\left(\cos ^{2} \theta_{a b} \cos ^{2} \theta_{b c}+\sin ^{2} \theta_{a b} \sin ^{2} \theta_{b c} / 2\right)-1>/ 2$

where we again used $<\cos \phi_{a b, b c}>=0$ and $<\cos ^{2} \phi_{a b, b c}>=1 / 2$. Eq. A3 can be rewritten as

$<P_{2}\left(\cos \theta_{a c}\right)>=<3 \cos ^{2} \theta_{a b} \cos ^{2} \theta_{b c}+3 / 2\left(1+\cos ^{2} \theta_{a b} \cos ^{2} \theta_{b c}-\cos ^{2} \theta_{a b}-\cos ^{2} \theta_{b c}\right)-1>/ 2$

$=<9 \cos ^{2} \theta_{a b} \cos ^{2} \theta_{b c}+3\left(1-\cos ^{2} \theta_{a b}-\cos ^{2} \theta_{b c}\right)>/ 4$

$=<3\left(\cos ^{2} \theta_{a b}-1\right) / 2><3\left(\cos ^{2} \theta_{b c}-1\right) / 2>$

$=<P_{2}\left(\cos \theta_{a b}\right)><P_{2}\left(\cos \theta_{b c}\right)>$

For small values of $\theta, \cos \theta \approx 1-\theta^{2} / 2$, and $P_{2}(\cos \theta) \approx 1-3 \theta^{2} / 2$; i.e. $<P_{2}\left(\cos \theta_{n}\right)>$ decays three times faster than $<\cos \theta_{n}>$, or:

$<P_{2}\left(\cos \theta_{n}\right)>=\exp (-3 n / L P)$ 


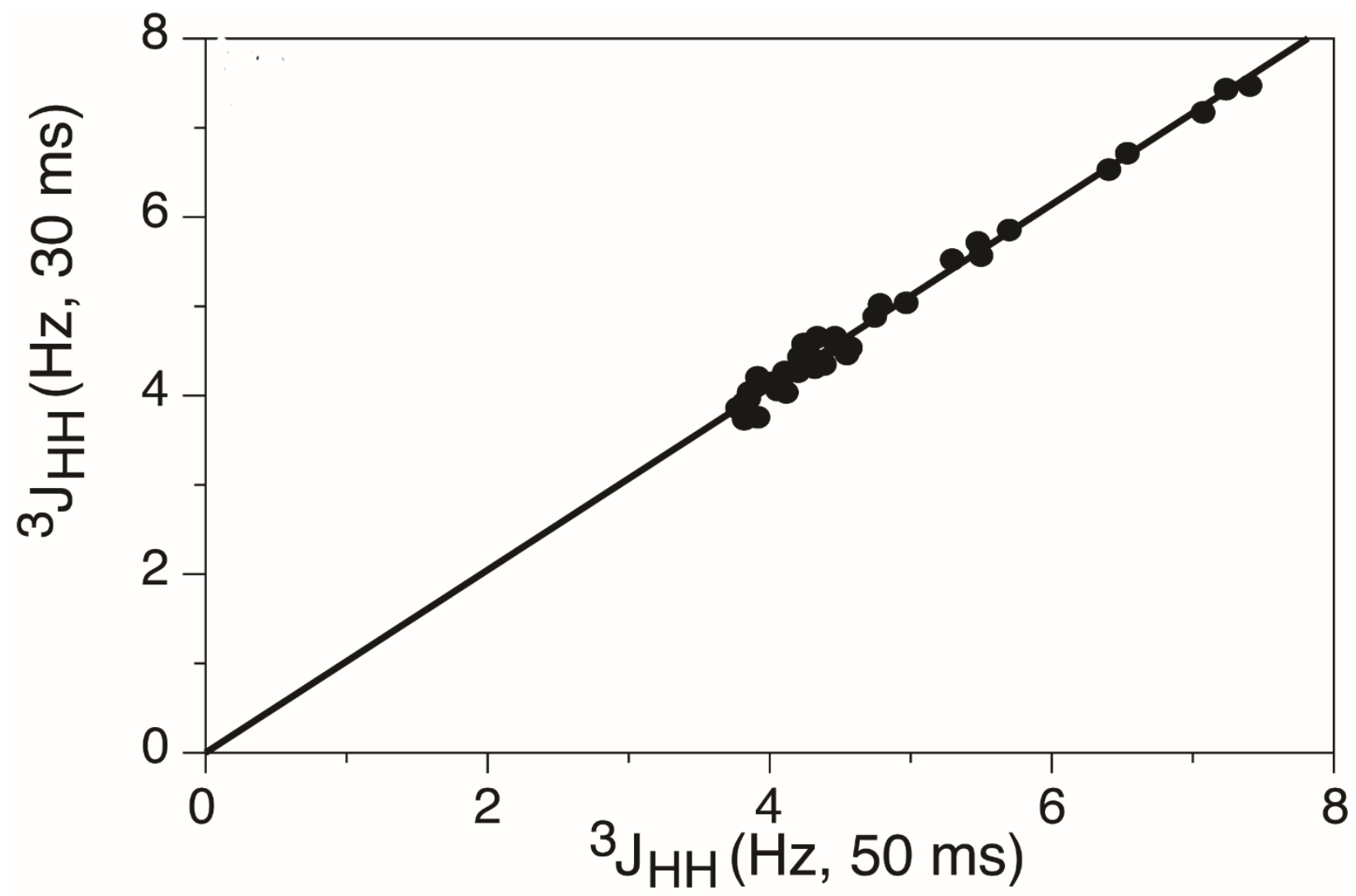

Figure S1. Correlation plot between the ${ }^{3} \mathrm{~J}_{\mathrm{HNH} \alpha}$ values measured with dephasing durations of 30 and $50 \mathrm{~ms}$. Data were collected at $900 \mathrm{MHz}$ using the ARTSY-J experiment, ${ }^{9}$ on fully protonated, ${ }^{15} \mathrm{~N}$-enriched $1 \mathrm{mM}$ MT at $\mathrm{pH} 6.3,35^{\circ} \mathrm{C}$ and are uncorrected for ${ }^{1} \mathrm{H}^{\alpha}$ spin flips. 


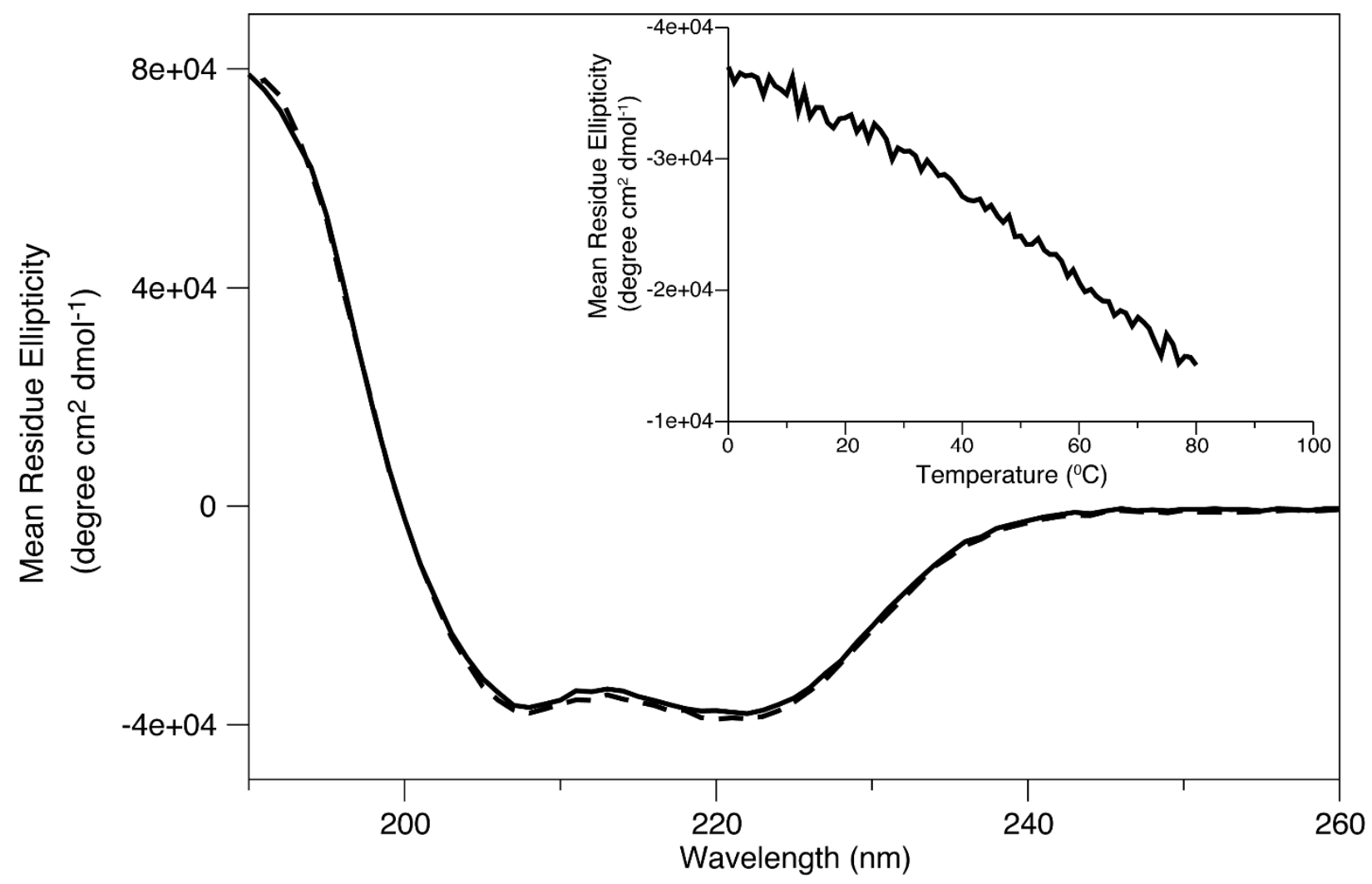

Figure S2. Circular dichroism spectra of the E68W MT domain $(10 \mu \mathrm{M})$ in $20 \mathrm{mM}$ sodium phosphate, $2 \mathrm{mM}$ EDTA, $\mathrm{pH}=6.3$ at $0{ }^{\circ} \mathrm{C}$ before (solid) and after (dashed) thermal melt measurements (inset), which report the mean residue ellipticity at $222 \mathrm{~nm}$ from $0^{\circ} \mathrm{C}$ to $80^{\circ} \mathrm{C}$. The helicity of the MT at $0{ }^{\circ} \mathrm{C}$ is $97 \%$ as calculated by the CDNN program. ${ }^{10}$ 

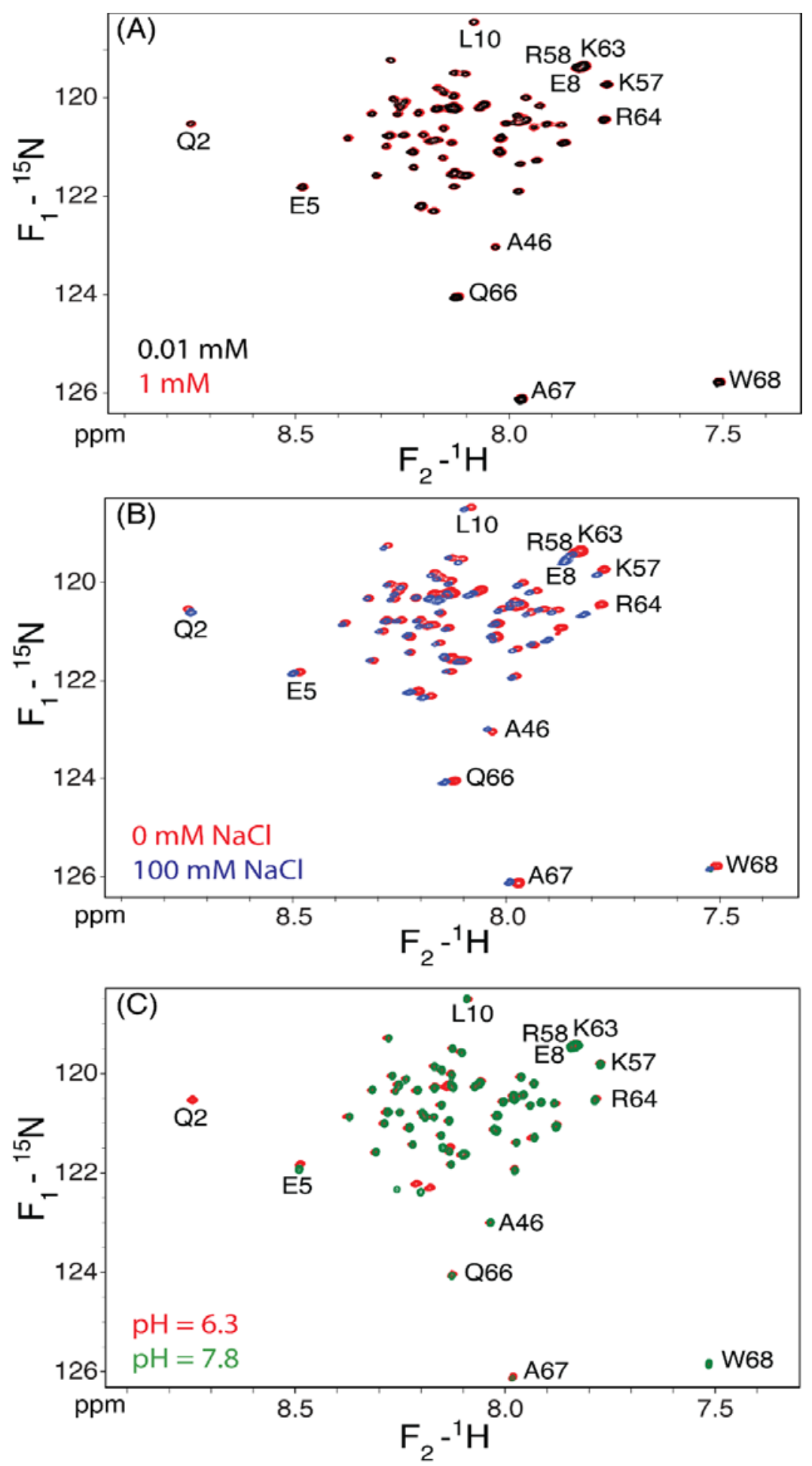

Figure S3. Comparison of $900 \mathrm{MHz}$ TROSY-HSQC spectra of perdeuterated ${ }^{15} \mathrm{~N}$-labeled E68W MT recorded under different conditions. (A) Spectra recorded in $20 \mathrm{mM}$ sodium phosphate, $2 \mathrm{mM}$ EDTA, $\mathrm{pH}=6.3$ at $20^{\circ} \mathrm{C}$ and concentrations of $10 \mu \mathrm{M}$ (black) and $1 \mathrm{mM}$ (red). (B) Spectra recorded in $20 \mathrm{mM}$ sodium phosphate, $2 \mathrm{mM}$ EDTA, $\mathrm{pH}=6.3$ at $20^{\circ} \mathrm{C}$ in the absence (red) and presence (blue) of $100 \mathrm{mM} \mathrm{NaCl}$. (C) Spectra in $20 \mathrm{mM}$ sodium phosphate, $2 \mathrm{mM}$ EDTA, at $20{ }^{\circ} \mathrm{C}$ in the absence of $100 \mathrm{mM} \mathrm{NaCl}$, at $\mathrm{pH} 6.3$ (red) and 7.8 (green). 


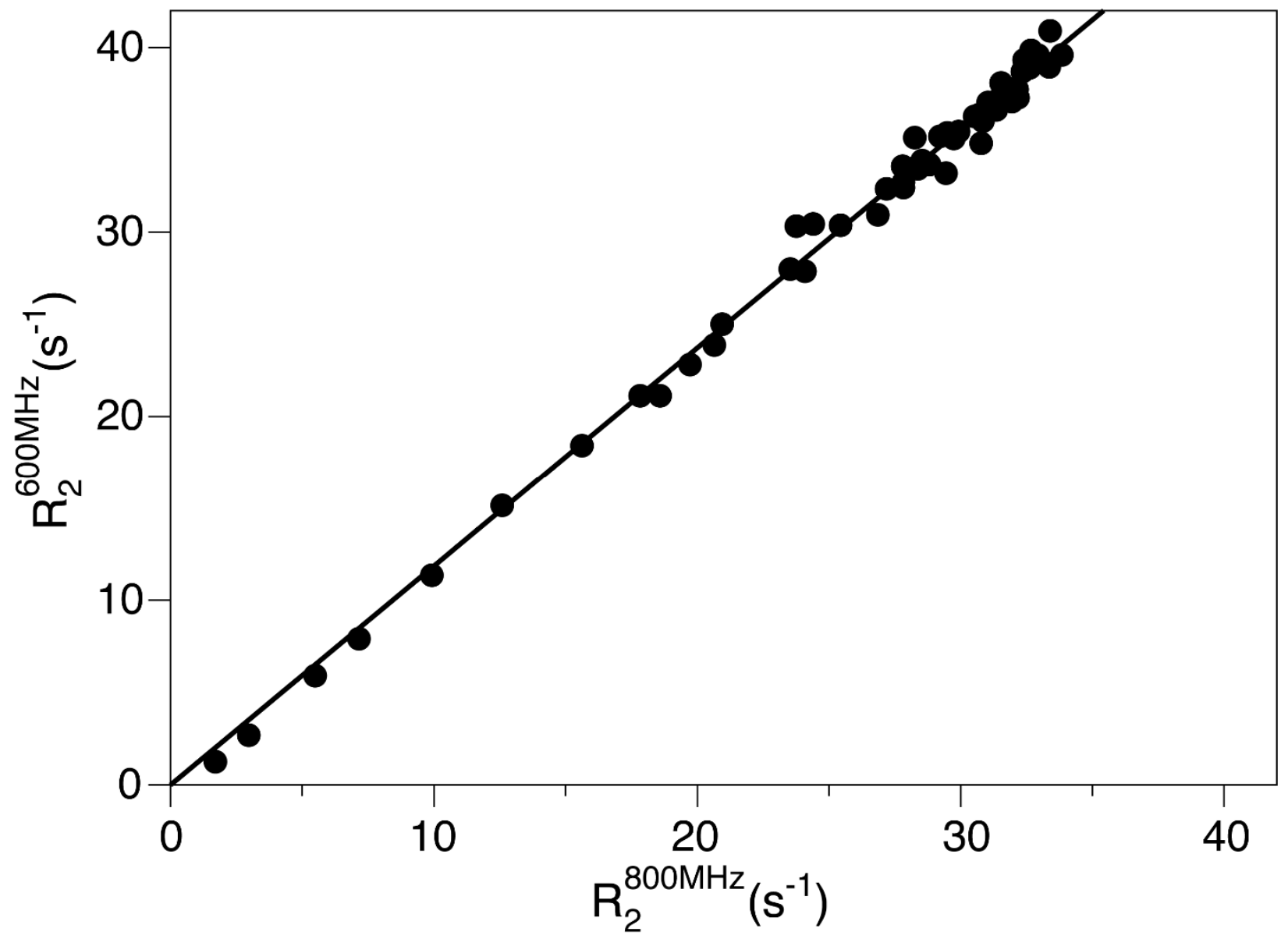

Figure S4. Correlation plot of ${ }^{15} \mathrm{~N} R_{1} \rho$ values measured at $14.1(600 \mathrm{MHz})$ and $18.8 \mathrm{~T}(800 \mathrm{MHz}$ ${ }^{1} \mathrm{H}$ frequency), using a $2.5 \mathrm{kHz}$ spin lock field. On-resonance $R_{1 \rho}$ values are derived from the measured values using the small, standard adjustment for off-resonance effects: $R_{1} \rho=\left(R \rho_{\rho}^{\prime}-\sin ^{2} \phi\right.$ $\left.R_{1}\right) / \cos ^{2} \phi$, where $R_{1 \rho}^{\prime}$ is the experimentally measured value, and $R_{1 \rho}$ is the on-resonance value. 

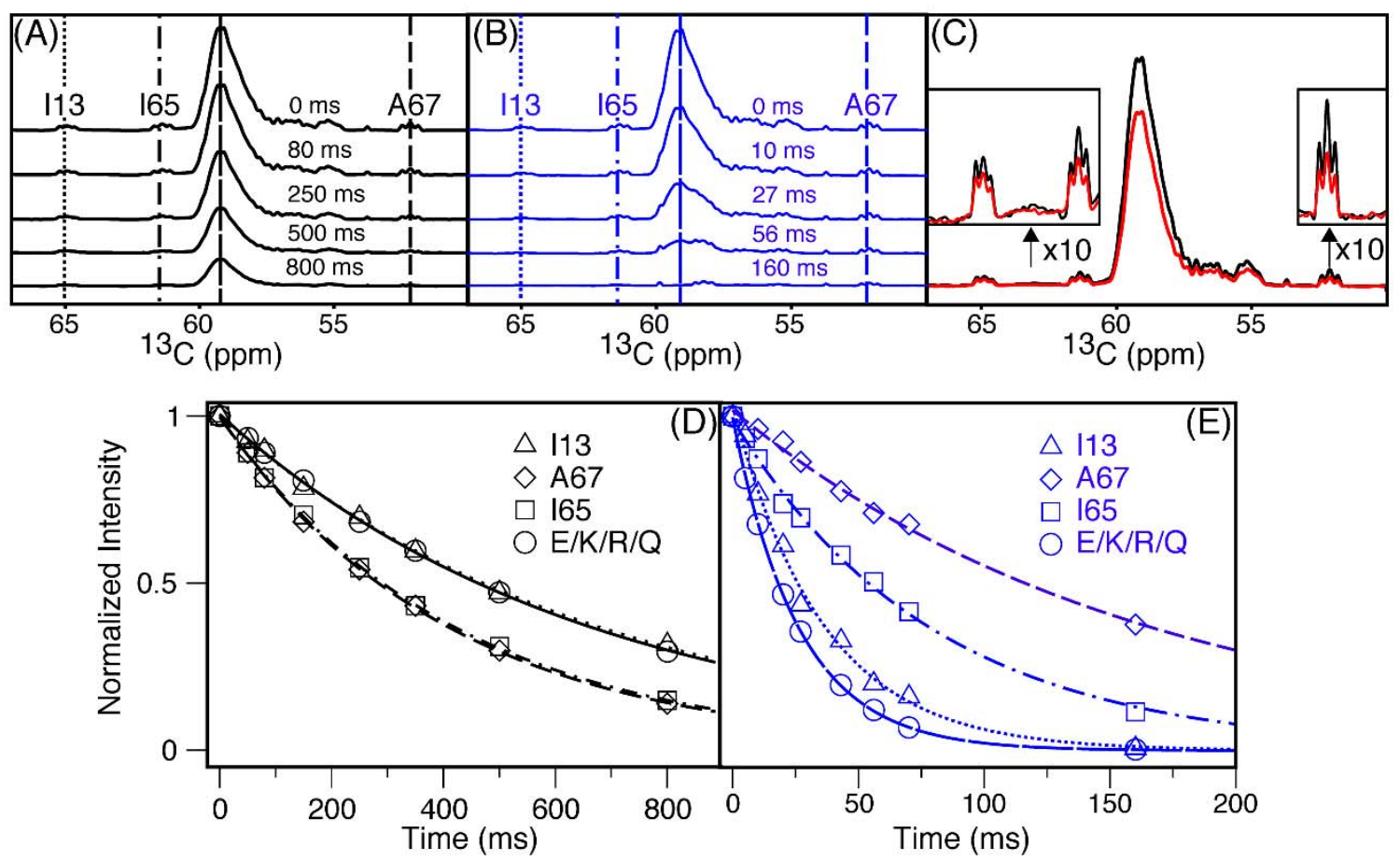

Figure S5. Direct observe ${ }^{13} \mathrm{C}^{\alpha}$ relaxation measurements of uniformly ${ }^{15} \mathrm{~N} /{ }^{13} \mathrm{C}$-enriched E68W MT at $14 \mathrm{~T}, 20^{\circ} \mathrm{C}$. (A) $R_{1}$ measurements were carried out with the Freeman-Hill modification ${ }^{11}$ of the regular inversion-recovery experiment, such as to allow for a two-parameter fit. Specifically, we fit the difference in signal between a $180^{\circ}$-T- $90^{\circ}$-Observe and a $90^{\circ}$-Observe experiment as a function of $\mathrm{T}$. The $180^{\circ}$ pulse is of the hyperbolic secant type ${ }^{12}$ centered at $100 \mathrm{ppm}$. Results of the fits are listed in Table S5. (B) $R_{1}$ measurement, using a $2.5 \mathrm{kHz}$ spin lock field with the carrier placed at $56 \mathrm{ppm}$. (C) Heteronuclear ${ }^{13} \mathrm{C}-\left\{{ }^{1} \mathrm{H}\right\}$ NOE measurement. The spectra result from 1024 transients with (black) and without (red) ${ }^{1} \mathrm{H}$ saturation during the $3 \mathrm{~s}$ delay between scans. The insets show the isolated signals of I13, I65 and A67 at 10 times higher scale. (D, E) Decay curves, taken at the positions indicated in $(\mathrm{A}, \mathrm{B})$. 

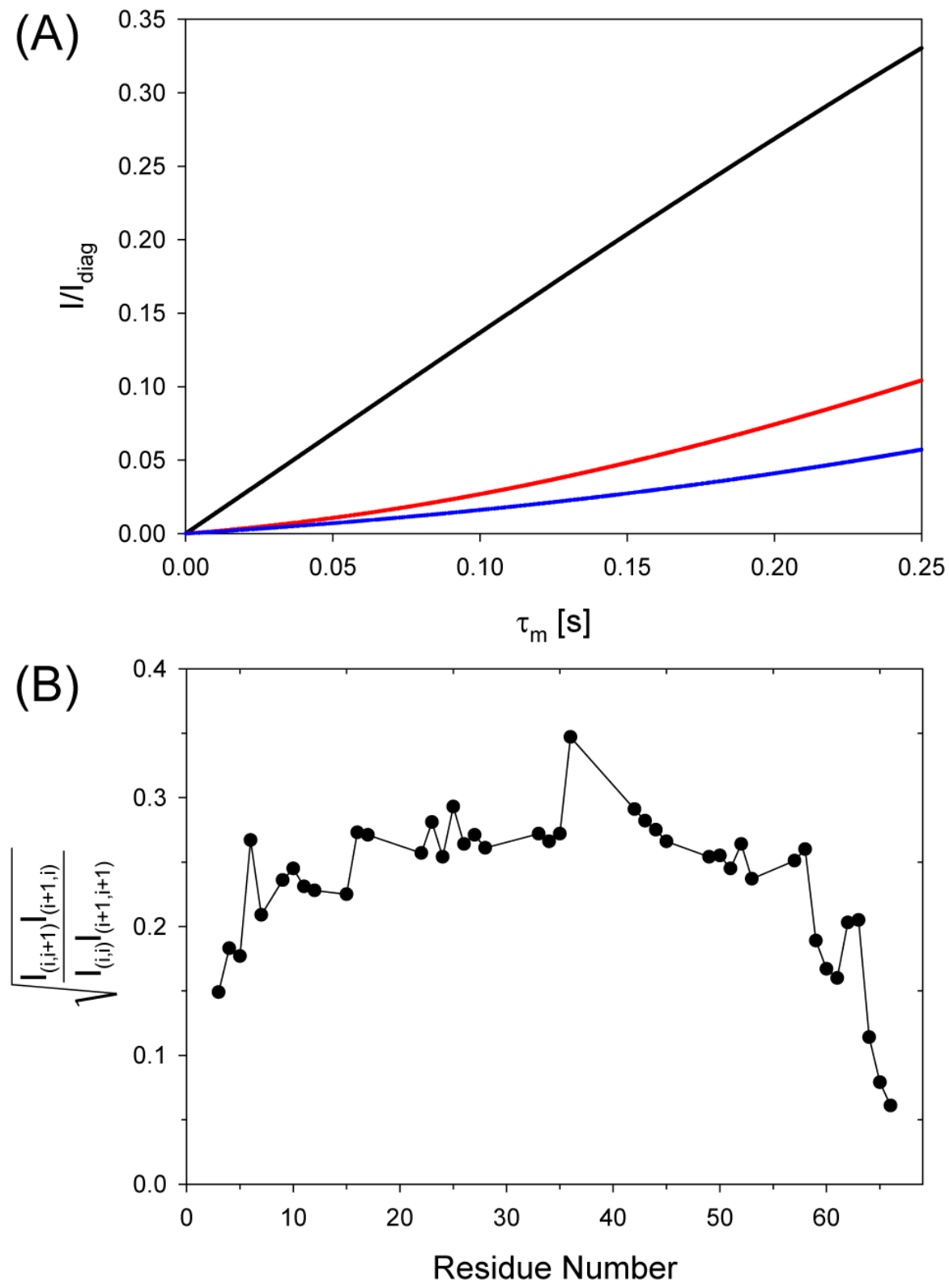

Figure S6. ${ }^{1} \mathrm{H}-{ }^{1} \mathrm{H}$ NOE intensities in the MT domain. (A) Calculated NOE intensities as a function of mixing time in an ideal $\alpha$-helix. Intensities are calculated for a system of seven sequential amide protons, using the spectral densities for an axially symmetric diffusion tensor with $\mathrm{D} / / / \mathrm{D} \perp$ $=7.6$ and $\tau_{\mathrm{m}}=10.3 \mathrm{~ns}$ (SI Text). The solid black line corresponds to the intensity of the $\mathrm{d}_{\mathrm{NN}}(4,4 \pm 1)$ cross peaks divided by the intensity of the spin 4 diagonal peak. Colored lines (red and blue) correspond to the respective intensities of the $\mathrm{d}_{\mathrm{NN}}(4,4 \pm 2)$ and $\mathrm{d}_{\mathrm{NN}}(4,4 \pm 3)$ cross peaks divided by the intensity of the spin-4 diagonal. (B) Normalized intensity of sequential $\mathrm{H}^{\mathrm{N}}-\mathrm{H}^{\mathrm{N}} \mathrm{NOE}$ cross peaks observed in the $250 \mathrm{~ms}$ mixing time 4D NOESY spectrum of MT, recorded at $900 \mathrm{MHz}{ }^{1} \mathrm{H}$ frequency, $20{ }^{\circ} \mathrm{C}$. Values are only shown for well-resolved peaks whose intensities could be accurately measured. 

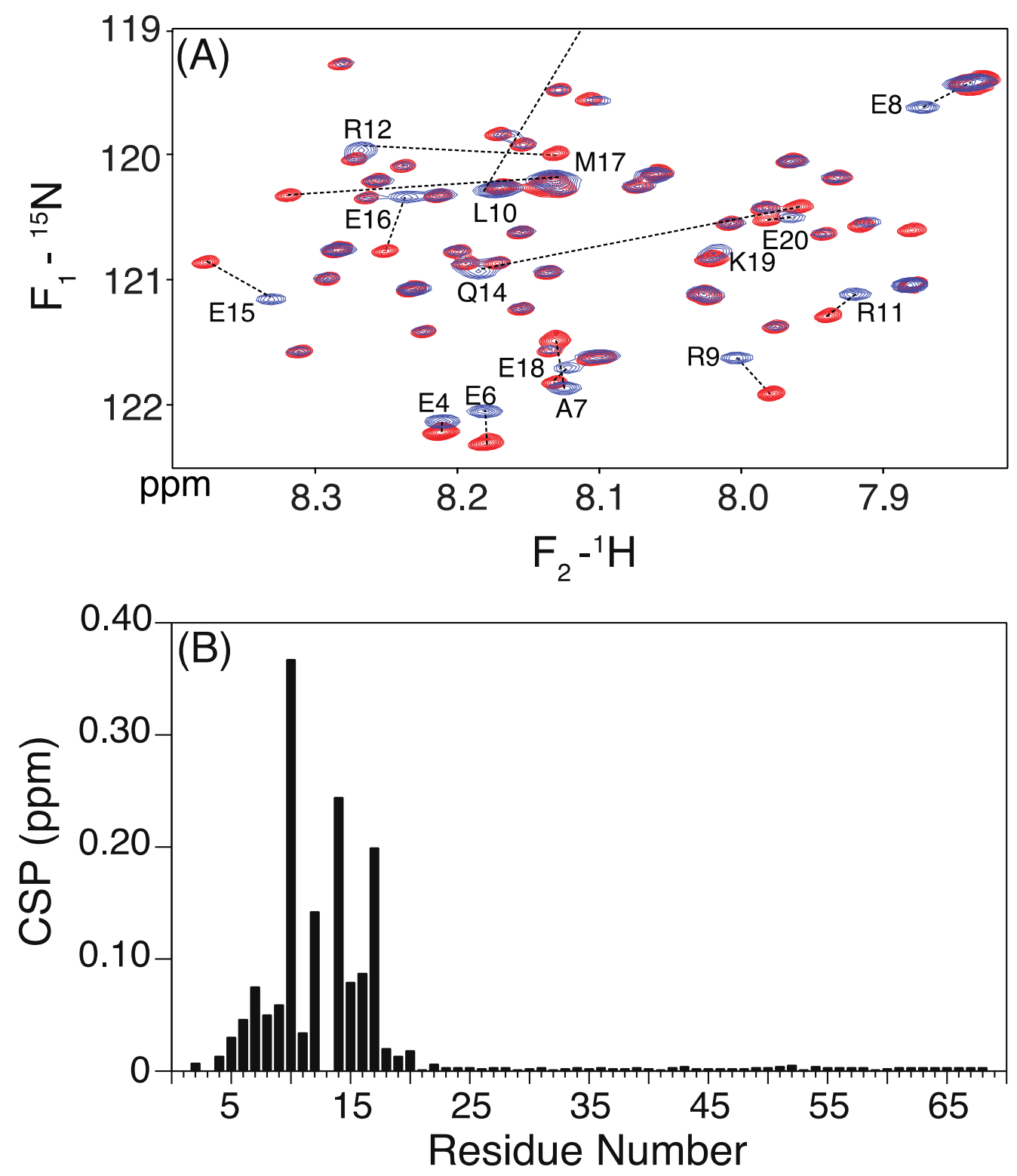

Figure S7. Chemical shift perturbation of E68W MT domain upon I13C mutation. (A) Overlay of the most crowded region of the E68W (red) and E68W/I13C (blue) ${ }^{1} \mathrm{H}^{-15} \mathrm{~N}$ TROSY-HSQC spectra of MT, recorded at $900 \mathrm{MHz}, 20{ }^{\circ} \mathrm{C}, 2 \mathrm{mM}$ EDTA, $\mathrm{pH}$ 6.3. Dashed lines connect resonance that are perturbed most; peak labels correspond to E68W/I13C MT (B) The chemical shift perturbation, calculated as CSP = $\sqrt{\left(\Delta \delta^{15} \mathrm{~N} / 5\right)^{2}+\left(\Delta \delta^{1} \mathrm{H}\right)^{2}}$, where $\Delta \delta$ denotes the chemical shift difference in units of ppm. 
(A)

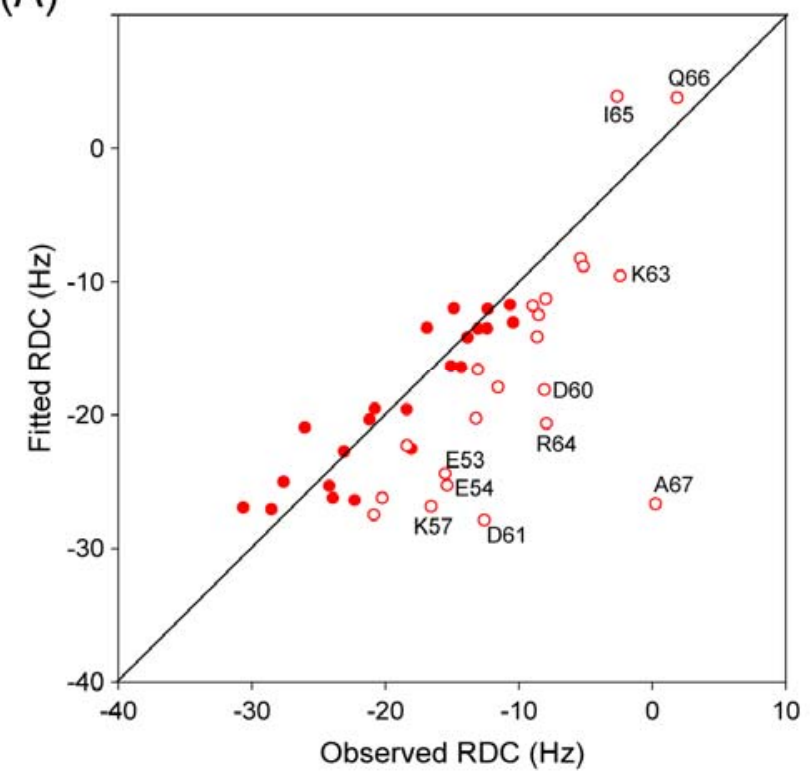

(B)

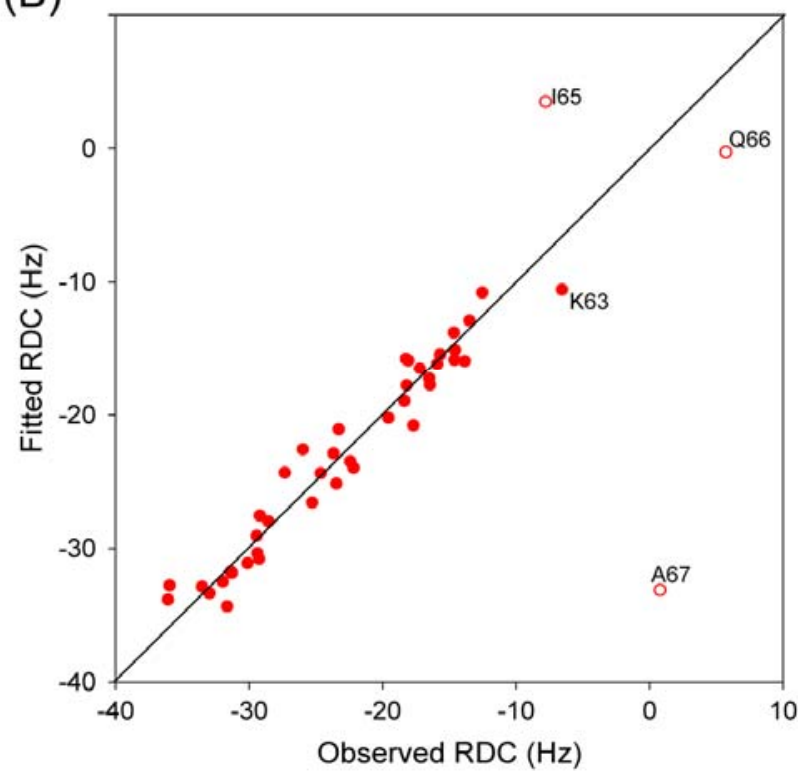

Figure S8. Plot of SVD best-fitted RDC data of DOTA-M8-Tm tagged I13C mutant of E68W MT in $20 \mathrm{mM}$ sodium phosphate, $2 \mathrm{mM}$ EDTA, $100 \mathrm{mM} \mathrm{NaCl}, 900 \mathrm{MHz}, 20^{\circ} \mathrm{C}$. (A) Alignment tensor best fit to the ${ }^{1} D_{\mathrm{NH}}$ of residues R24-E45. (B) Alignment tensor best fit to the ${ }^{1} D_{\mathrm{NH}}$ of residues R24-R64, after upscaling of ${ }^{1} D_{\mathrm{NH}}$ by $\exp [(+(i-13) / 49.75]$. Open symbols in each panel correspond to residues not included in the SVD fit.

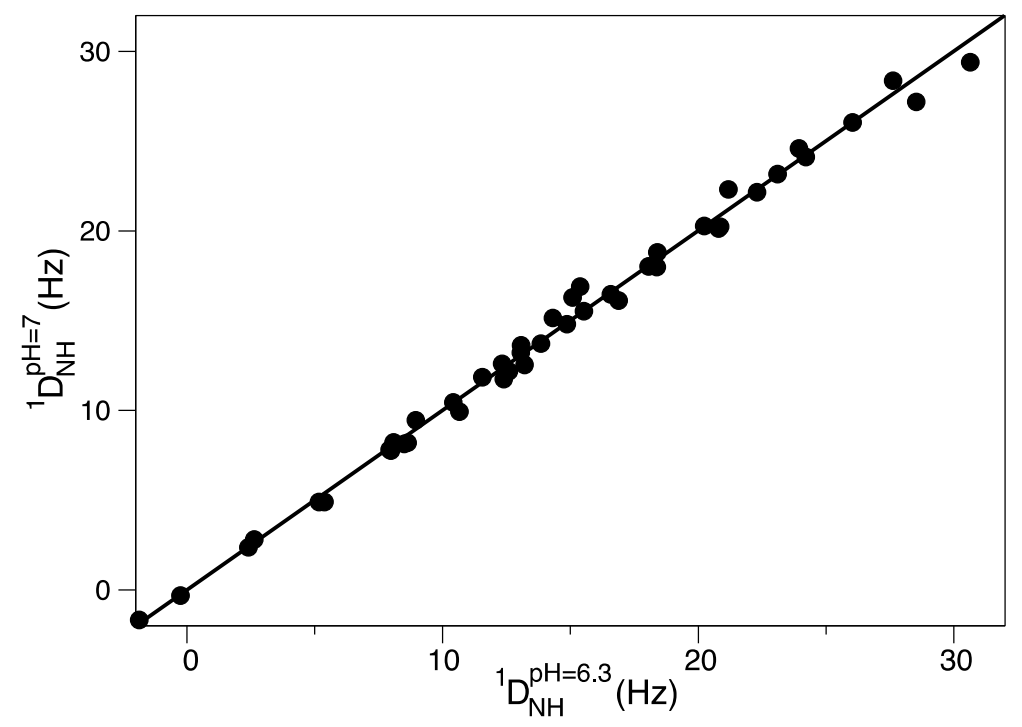

Figure S9. Correlation plot of the RDC data of DOTA-M8-Tm tagged I13C mutant of E68W MT in $20 \mathrm{mM}$ sodium phosphate, $2 \mathrm{mM}$ EDTA, $100 \mathrm{mM} \mathrm{NaCl}, 900 \mathrm{MHz}, 20{ }^{\circ} \mathrm{C}$ at $\mathrm{pH} 6.3$ versus $\mathrm{pH}$ 7.0. The pairwise RMSD between the two sets of values is $0.3 \mathrm{~Hz}$. 
Table S1. Chemical shifts and ${ }^{3} \mathrm{~J}_{\mathrm{HNH} \alpha}$ values of the native MT domain as well as chemical shifts of the I13C/E68W double mutant, recorded in $20 \mathrm{mM}$ sodium phosphate, $2 \mathrm{mM}$ EDTA, $\mathrm{pH}=6.3$. ${ }^{1} \mathrm{H}$ and ${ }^{15} \mathrm{~N}$ chemical shifts are at $20{ }^{\circ} \mathrm{C} ;{ }^{13} \mathrm{C}^{\alpha}$ and ${ }^{13} \mathrm{C}^{\beta}$ shifts are at $35{ }^{\circ} \mathrm{C}$.

\begin{tabular}{|c|c|c|c|c|c|c|c|}
\hline Residue & $\begin{array}{l}{ }^{1} \mathrm{H} \\
\text { (ppm) }\end{array}$ & $\begin{array}{l}{ }^{15} \mathrm{~N} \\
\text { (ppm) }\end{array}$ & $\begin{array}{l}{ }^{1} \mathrm{H} \\
(\mathrm{ppm})^{\mathrm{a}}\end{array}$ & $\begin{array}{l}{ }^{15} \mathrm{~N} \\
(\mathrm{ppm})^{\mathrm{a}}\end{array}$ & ${ }^{13} C^{\alpha}(p p m)$ & $\begin{array}{l}{ }^{13} \mathrm{C}^{\beta} \\
\text { (ppm) }\end{array}$ & $\begin{array}{l}{ }^{3} \mathrm{~J}_{\mathrm{HNH} \alpha} \\
(\mathrm{Hz})\end{array}$ \\
\hline Q2 & 8.79 & 120.01 & 8.80 & 119.99 & & & \\
\hline Q3 & 8.18 & 119.71 & 8.19 & 119.69 & & & \\
\hline E4 & 8.26 & 121.70 & 8.25 & 121.63 & 57.56 & 27.81 & 6.1 \\
\hline E5 & 8.54 & 121.30 & 8.52 & 121.18 & 57.80 & 28.93 & 5.1 \\
\hline E6 & 8.23 & 121.78 & 8.23 & 121.55 & 58.53 & 28.75 & 4.9 \\
\hline A7 & 8.18 & 121.00 & 8.17 & 121.37 & 54.71 & 17.31 & 4.2 \\
\hline E8 & 7.89 & 118.95 & 7.92 & 119.15 & 58.21 & 28.45 & \\
\hline R9 & 8.03 & 121.40 & 8.05 & 121.14 & 59.24 & 29.36 & 4.1 \\
\hline L10 & 8.14 & 118.02 & 8.22 & 119.80 & 57.28 & 40.47 & 4.2 \\
\hline R11 & 7.99 & 120.78 & 7.97 & 120.64 & 58.87 & 28.99 & 4.3 \\
\hline R12 & 8.18 & 119.49 & 8.32 & 119.46 & 58.89 & 28.93 & 4.4 \\
\hline I13 (C13) & 7.93 & 120.12 & (8.22) & (118.24) & 64.46 & 37.36 & 4.4 \\
\hline Q14 & 8.01 & 119.92 & 8.23 & 120.44 & 58.55 & 27.26 & 4.3 \\
\hline E15 & 8.43 & 120.35 & 8.38 & 120.66 & 59.04 & 28.78 & 4.1 \\
\hline E16 & 8.30 & 120.27 & 8.29 & 119.84 & 58.83 & 28.52 & \\
\hline M17 & 8.37 & 119.82 & 8.17 & 119.67 & 58.45 & 31.75 & 4.2 \\
\hline E18 & 8.18 & 121.31 & 8.17 & 121.22 & 58.45 & 28.52 & 4.2 \\
\hline K19 & 8.07 & 120.34 & 8.07 & 120.28 & 59.27 & 31.50 & \\
\hline E20 & 8.03 & 120.00 & 8.02 & 120.01 & 58.44 & 28.44 & \\
\hline R21 & 8.16 & 121.13 & 8.16 & 121.13 & 59.23 & 28.86 & \\
\hline K22 & 8.16 & 119.07 & 8.15 & 119.08 & 58.77 & 31.26 & 4.4 \\
\hline R23 & 7.96 & 120.06 & 7.96 & 120.05 & 58.72 & 29.04 & 4.4 \\
\hline R24 & 8.22 & 119.34 & 8.22 & 119.35 & 58.86 & 29.11 & 4.5 \\
\hline E25 & 8.25 & 120.27 & 8.25 & 120.28 & 58.86 & 28.84 & 4.6 \\
\hline E26 & 8.34 & 120.49 & 8.34 & 120.50 & 58.89 & 28.63 & 4.4 \\
\hline D27 & 8.36 & 121.07 & 8.36 & 121.09 & 57.07 & 40.41 & 3.9 \\
\hline K28 & 8.24 & 120.37 & 8.25 & 120.39 & 58.85 & 28.48 & 4.3 \\
\hline Q29 & 8.18 & 118.99 & 8.18 & 119.00 & 58.47 & 27.35 & 4.6 \\
\hline R30 & 8.15 & 121.12 & 8.15 & 121.12 & & & \\
\hline R31 & 8.18 & 119.71 & 8.18 & 119.70 & 59.06 & 29.06 & \\
\hline R32 & 8.20 & 119.43 & 8.20 & 119.43 & 58.86 & 29.12 & 4.3 \\
\hline K33 & 8.03 & 120.87 & 8.03 & 120.88 & 58.75 & 31.18 & 3.9 \\
\hline E34 & 8.29 & 119.59 & 8.29 & 119.60 & 58.92 & 28.63 & 4.2 \\
\hline E35 & 8.22 & 120.36 & 8.22 & 120.37 & 58.79 & 28.52 & 4.5 \\
\hline
\end{tabular}




\begin{tabular}{|c|c|c|c|c|c|c|c|}
\hline E36 & 8.19 & 121.06 & 8.19 & 121.07 & 58.81 & 28.67 & 4.3 \\
\hline E37 & 8.31 & 119.84 & 8.31 & 119.86 & 59.05 & 28.65 & 3.9 \\
\hline R38 & 8.07 & 120.34 & 8.07 & 120.34 & 59.13 & 29.05 & \\
\hline R39 & 8.17 & 119.78 & 8.18 & 119.80 & & & \\
\hline M40 & 8.33 & 118.77 & 8.33 & 118.78 & & & \\
\hline K41 & 8.07 & 120.65 & 8.07 & 120.65 & & & \\
\hline L42 & 7.99 & 120.13 & 7.99 & 120.14 & 57.54 & 40.71 & 4.3 \\
\hline E43 & 8.27 & 120.90 & 8.27 & 120.92 & 58.83 & 28.71 & 4.7 \\
\hline M44 & 8.32 & 119.53 & 8.32 & 119.54 & 59.14 & 31.45 & 4.8 \\
\hline E45 & 8.21 & 120.73 & 8.21 & 120.74 & 58.56 & 28.60 & \\
\hline A46 & 8.09 & 122.50 & 8.09 & 122.50 & 54.44 & 17.18 & 4.0 \\
\hline K47 & 8.03 & 119.93 & 8.03 & 119.94 & 58.46 & 31.40 & 4.7 \\
\hline R48 & 8.06 & 120.05 & 8.06 & 120.06 & 58.49 & 28.99 & 4.2 \\
\hline K49 & 8.12 & 119.76 & 8.12 & 119.76 & 58.61 & 31.39 & 4.4 \\
\hline Q50 & 7.98 & 119.68 & 7.98 & 119.69 & 58.68 & 28.68 & 4.5 \\
\hline E51 & 8.31 & 119.70 & 8.31 & 119.72 & 58.68 & 28.62 & 4.5 \\
\hline E52 & 8.19 & 120.43 & 8.19 & 120.45 & 58.55 & 28.73 & 4.8 \\
\hline E53 & 8.21 & 120.13 & 8.21 & 120.13 & 58.59 & 28.71 & 4.6 \\
\hline E54 & 8.26 & 119.83 & 8.26 & 119.84 & 58.42 & 28.67 & 4.7 \\
\hline R55 & 8.07 & 120.59 & 8.08 & 120.62 & & & \\
\hline K56 & 8.01 & 119.58 & 8.01 & 119.56 & 58.13 & 31.52 & 5.2 \\
\hline K57 & 7.83 & 119.30 & 7.83 & 119.30 & 57.98 & 31.57 & 5.9 \\
\hline R58 & 7.89 & 118.95 & 7.88 & 118.91 & & & \\
\hline E59 & 8.22 & 119.78 & 8.22 & 119.78 & 57.70 & 29.04 & 5.7 \\
\hline D60 & 8.35 & 120.28 & 8.34 & 120.27 & 55.49 & 40.27 & 5.3 \\
\hline D61 & 8.28 & 120.62 & 8.28 & 120.59 & 55.54 & 40.27 & 5.6 \\
\hline E62 & 8.10 & 119.64 & 8.11 & 119.66 & 57.32 & 29.05 & 5.8 \\
\hline K63 & 7.90 & 119.18 & 7.89 & 118.94 & 56.46 & 31.55 & 6.4 \\
\hline R64 & 7.89 & 120.44 & 7.83 & 120.01 & 56.33 & 29.64 & 6.8 \\
\hline 165 & 8.02 & 120.95 & 7.93 & 120.54 & 61.05 & 37.59 & 7.7 \\
\hline Q66 & 8.28 & 123.61 & 8.18 & 123.56 & 55.31 & 28.68 & 7.4 \\
\hline A67 & 8.23 & 125.85 & 8.03 & 125.63 & 52.04 & 18.68 & 7.0 \\
\hline E68 (W68) & 7.95 & 125.34 & (7.57) & $(125.34)$ & 57.54 & 30.36 & 7.7 \\
\hline
\end{tabular}


Table S2. Backbone RDCs and isotropic $J$ values $(\mathrm{Hz})$ of the W68 MT domain in $20 \mathrm{mM}$ sodium phosphate, $2 \mathrm{mM}$ EDTA, $100 \mathrm{mM} \mathrm{NaCl}, \mathrm{pH}=6.3$ measured in the absence and presence of 13 $\mathrm{mg} / \mathrm{mL} \mathrm{Pf} 1$ at $20^{\circ} \mathrm{C}$. Experimental uncertainties in the measured couplings are $0.5 \mathrm{~Hz}\left({ }^{1} \mathrm{D}_{\mathrm{NH}}\right), 0.2$ $\mathrm{Hz}\left({ }^{1} \mathrm{D}_{\mathrm{NC}^{\prime}}\right) 0.4 \mathrm{~Hz}\left({ }^{2} \mathrm{D}_{\mathrm{C}^{\prime} \mathrm{H}}\right), 0.3 \mathrm{~Hz}\left({ }^{1} J_{\mathrm{NH}}\right), 0.1 \mathrm{~Hz}\left({ }^{1} J_{\mathrm{NC}^{\prime}}\right)$ and $0.2 \mathrm{~Hz}\left({ }^{2} J_{\mathrm{C}^{\prime} \mathrm{H}}\right)$. Couplings are listed according to the residue number of the amide ${ }^{1} \mathrm{H}$ or ${ }^{15} \mathrm{~N}$ and ignore the negative sign of the ${ }^{15} \mathrm{~N}$ gyromagnetic ratio.

\begin{tabular}{|c|c|c|c|c|c|c|c|}
\hline es & ${ }^{1} \mathrm{D}_{\mathrm{NH}}$ & ${ }^{1} D_{N H^{a}}$ & ${ }^{1} \mathrm{D}_{\mathrm{NC}}$ & ${ }^{2} D_{C^{\prime} H}$ & ${ }^{1} J_{\mathrm{NH}}$ & ${ }^{2} J_{C^{\prime} H}$ & ${ }^{1} J_{\mathrm{N}}$ \\
\hline $\mathrm{Q} 2$ & -7.3 & 2.2 & 0.4 & -0.2 & 92.9 & 3.8 & \\
\hline 4 & -11.4 & -2.1 & -0.6 & -4.2 & 93.0 & 4.2 & \\
\hline 5 & -9.9 & 1.0 & 1.5 & 1.0 & 93.3 & 4.1 & \\
\hline & -19.3 & -6.6 & 0.9 & -1.1 & 92.9 & 4.3 & \\
\hline 7 & -25.2 & -13.8 & 0.9 & -2.8 & 92.9 & 4.4 & \\
\hline 9 & -22.1 & -7.9 & 1.5 & -0.5 & 93.2 & 4.3 & 4 \\
\hline 10 & -24.9 & -13.5 & 1.4 & -1.5 & 93.3 & 4.4 & \\
\hline 11 & -25.6 & -11.4 & 0.4 & -3.7 & 93.1 & 4.4 & \\
\hline 12 & -21.9 & -6.1 & & & 93.5 & & \\
\hline 3 & -23.7 & -10.8 & 1.3 & -1.3 & 93.0 & 4.0 & \\
\hline 214 & -28.5 & -15.4 & 1.6 & -2.5 & 92.7 & 4.3 & 4 \\
\hline 15 & -26.0 & -8.8 & 0.6 & -2.7 & 92.5 & 4.1 & 14 \\
\hline 6 & -22.2 & -6.5 & 2.1 & 0.3 & 93.1 & 4.2 & 15 \\
\hline И17 & -27.0 & -13.7 & 1.1 & -1.4 & 93.4 & 4.2 & 15 \\
\hline 18 & -30.5 & -16.1 & 1.2 & -4.1 & 93.5 & 4.0 & 5 \\
\hline 22 & -30.5 & -13.6 & 0.8 & -3.5 & 93.7 & 4.2 & 15 \\
\hline 23 & -27.3 & -8.8 & 2.0 & -0.1 & 93.0 & 4.3 & 15 \\
\hline 224 & -28.5 & -15.0 & 1.1 & -2.5 & 93.9 & 4.2 & 5 \\
\hline 25 & -33.7 & -18.9 & 0.9 & -4.2 & 92.9 & 4.2 & 15 \\
\hline 6 & -29.4 & -10.2 & & & 92.8 & & \\
\hline 27 & -28.7 & -12.0 & 2.2 & 0.1 & 93.4 & 4.4 & \\
\hline K28 & -31.9 & -18.6 & 1.0 & -3.8 & 93.7 & 4.1 & 15. \\
\hline 29 & -32.0 & -17.9 & 1.0 & -4.2 & 93.4 & 4.1 & 14 \\
\hline 32 & -28.3 & -20.6 & 1.5 & -3.9 & 93.2 & 4.1 & 14 \\
\hline K33 & -32.6 & -14.7 & 0.9 & -3.9 & 92.7 & 4.3 & 15 \\
\hline 34 & -32.4 & -12.1 & & & 92.7 & & \\
\hline 35 & -30.8 & -17.7 & 1.0 & -1.8 & 93.0 & 4.3 & -3 \\
\hline$E 37$ & -31.3 & -13.6 & & & 91.9 & & \\
\hline R39 & -31.6 & & 0.6 & -2.0 & 92.6 & 4.4 & 14. \\
\hline M40 & -29.9 & -17.5 & & & 93.2 & 4.3 & 14 \\
\hline L42 & -27.5 & -18.0 & 1.3 & -2.9 & 92.9 & 4.4 & 15 \\
\hline E43 & -32.0 & -20.3 & 1.4 & -3.3 & 93.3 & 4.3 & \\
\hline M44 & -28.9 & -12.1 & & & 93.1 & & \\
\hline E45 & -30.6 & -11.9 & 2.4 & 0.7 & 93.4 & 4.4 & \\
\hline A46 & -34.6 & -18.7 & 1.2 & -2.9 & 92.9 & 4.2 & \\
\hline R48 & -30.0 & -11.0 & 1.3 & 0.0 & 92.9 & 4.2 & \\
\hline K49 & -33.7 & -14.1 & & & 93.0 & & \\
\hline
\end{tabular}




$\begin{array}{llllllll}\text { Q50 } & -34.1 & -20.4 & 1.3 & -2.9 & 92.5 & 4.3 & 15.0 \\ \text { E51 } & -28.5 & -15.7 & & & 93.6 & & \\ \text { E52 } & -30.4 & -9.3 & 2.3 & 0.2 & 92.9 & 4.3 & 15.2 \\ \text { E53 } & -33.5 & -17.3 & 1.2 & -0.9 & 93.3 & 4.2 & 14.9 \\ \text { E54 } & -29.7 & -19.1 & 1.4 & -3.4 & 93.0 & 4.3 & 15.2 \\ \text { K56 } & -25.5 & -10.6 & 2.3 & 0.9 & 92.9 & 4.3 & 15.0 \\ \text { K57 } & -29.9 & -17.8 & 1.0 & -2.6 & 93.1 & 4.3 & 15.0 \\ \text { E59 } & -31.8 & -6.4 & 1.5 & 0.0 & 93.1 & 4.3 & 15.1 \\ \text { D60 } & -25.5 & -11.6 & 1.2 & -0.2 & 93.3 & 4.2 & 15.3 \\ \text { D61 } & -25.1 & -14.8 & 1.1 & -2.2 & 93.3 & 4.1 & 15.2 \\ \text { E62 } & -28.7 & -7.1 & 0.6 & -1.6 & 93.0 & 4.0 & 15.2 \\ \text { R64 } & -19.5 & -8.4 & 0.7 & -0.5 & 93.1 & 4.1 & 15.4 \\ \text { I65 } & -21.3 & -2.5 & 0.3 & -0.1 & 93.1 & 4.2 & 15.3 \\ \text { Q66 } & -22.9 & 2.7 & 0.3 & 0.9 & 92.9 & 4.1 & 14.9 \\ \text { A67 } & -16.8 & 1.1 & 0.3 & 1.4 & 92.6 & 4.0 & 15.1\end{array}$

a measurements in the absence of $100 \mathrm{mM} \mathrm{NaCl}$ 
Table S3. Experimental validation statistics for different structural representations of MT. ${ }^{a}$

\begin{tabular}{|l|l|l|}
\hline & Ideal $\alpha$-helix & Exp. structure \\
\hline$Q_{\text {free }}(\%)^{\mathrm{b}}$ & $\begin{array}{l}21.1 / 27.4 / 13.9 / 17.7 \\
(32.0)^{\mathrm{b}}\end{array}$ & - \\
\hline $\mathrm{Q}_{\text {free }}(\%)^{\mathrm{c}}$ & $\begin{array}{l}15.6 / 16.5 / 13.9 / 15.9 \\
(27.1)^{\mathrm{c}}\end{array}$ & $\begin{array}{l}12.9 / 13.8 / 12.5 / 12.0 \\
(22.8)^{\mathrm{c}, \mathrm{d}}\end{array}$ \\
\hline $\mathrm{Q}_{\text {fit }}(\%)^{\mathrm{e}}$ & - & $\begin{array}{l}10.4 / 7.1 / 12.2 / 11.9(15.9) \\
\mathrm{e}\end{array}$ \\
\hline
\end{tabular}

${ }^{\mathrm{a}}$ For residues E6-K63.

${ }^{\mathrm{b}}$ reported Q factors are for all/ ${ }^{1} \mathrm{D}_{\mathrm{NH}} /{ }^{1} \mathrm{D}_{\mathrm{NC}} /{ }^{2} \mathrm{D}_{\mathrm{C}^{\prime} \mathrm{H}}$ values, respectively, measured in the sample containing $100 \mathrm{mM} \mathrm{NaCl}$, with $D_{\mathrm{a}}=15.62 \mathrm{~Hz}$ and $R=0.16$ used for calculating the denominator of the $\mathrm{Q}$ factor and all couplings normalized to the $21,585 \mathrm{~Hz}{ }^{15} \mathrm{~N}-{ }^{1} \mathrm{H}$ dipolar interaction strength, i.e., ${ }^{1} \mathrm{D}_{\mathrm{NC}^{\prime}}$ and ${ }^{2} \mathrm{D}_{\mathrm{C}^{\prime} \mathrm{H}}$ upscaled by 8.26 and 3.10, respectively from their experimental values. The $\mathrm{Q}$ factor for the ${ }^{1} \mathrm{D}_{\mathrm{NH}}$ values measured from the sample without the addition of $100 \mathrm{mM} \mathrm{NaCl}$ are given in parenthesis.

${ }^{\mathrm{c}}$ same as $\mathrm{b}$ but the $\mathrm{Q}$ factors are reported for all/ ${ }^{1} \mathrm{D}_{\mathrm{NH}} /{ }^{1} \mathrm{D}_{\mathrm{NC}} /{ }^{2} \mathrm{D}_{\mathrm{C}^{\prime} \mathrm{H}}$ values, respectively, after upscaling by the inverse of the ${ }^{15} \mathrm{~N} R_{2}$ rate (see main text), and with a $\mathrm{D}_{\mathrm{a}}=16.88 \mathrm{~Hz}$ and $R=0.16$.

${ }^{\mathrm{d}}$ Free $\mathrm{Q}$ factors for the RDC-refined structure (see main text).

${ }^{\mathrm{e}} \mathrm{Q}$ factors reported for the ensemble of lowest energy final RDC-refined structures, for which all couplings of residues E6 to K63 were used. 
Table S4. ${ }^{15} \mathrm{~N}$ relaxation rates of the MT domain in $20 \mathrm{mM}$ sodium phosphate, $2 \mathrm{mM}$ EDTA, $\mathrm{pH}=6.3,20{ }^{\circ} \mathrm{C}$, measured at 600 and $800 \mathrm{MHz}{ }^{1} \mathrm{H}$ frequency, and best fit dynamics parameters obtained using Modelfree 4 while restraining the diffusion tensor to be axially symmetric with $\tau_{\mathrm{m}}$ $=10.3 \mathrm{~ns}$, and $\rho=7.5$. TROSY $R_{2}$ values $\left(R_{2, \mathrm{TR}}\right)$ correspond to ${ }^{15} \mathrm{~N}$ Hahn-echo decay rates. All $R_{1}$ and $R_{2}$ rates are in units of $\mathrm{s}^{-1}$, and errors are estimated at $\pm 3 \%$

\begin{tabular}{|c|c|c|c|c|c|c|c|}
\hline \multicolumn{8}{|c|}{$\mathrm{NOE}^{\circ}$} \\
\hline \multicolumn{8}{|l|}{ E4 } \\
\hline \multicolumn{8}{|l|}{ E5 } \\
\hline E6 & 0.86 & 1.10 & 27.9 & 24.1 & 0.35 & 1000 & 0.71 \\
\hline R9 & 0.79 & 0.99 & 30.9 & 26.9 & 0.39 & 1000 & 0.75 \\
\hline L10 & 0.77 & 0.99 & 30.4 & 24.4 & 0.42 & 980 & 0.77 \\
\hline R11 & 0.76 & 0.96 & 32.4 & 27.8 & 0.42 & 1100 & 0.76 \\
\hline R12 & 0.76 & 0.94 & 32.7 & 27.8 & 0.47 & 1100 & 0.78 \\
\hline I13 & 0.73 & 0.93 & 32.3 & 27.2 & 0.46 & 1000 & 0.79 \\
\hline Q14 & 0.70 & 0.90 & 33.7 & 28.8 & 0.50 & 1100 & 0.81 \\
\hline E15 & 0.69 & 0.88 & 35.1 & 29.7 & 0.48 & 1100 & 0.80 \\
\hline E16 & 0.70 & 0.90 & 33.2 & 29.4 & 0.49 & 1100 & 0.81 \\
\hline M17 & 0.67 & 0.87 & 35.2 & 29.2 & 0.51 & 1100 & 0.82 \\
\hline E18 & 0.68 & 0.86 & 34.8 & 30.8 & 0.50 & 1100 & 0.82 \\
\hline E20 & 0.61 & 0.82 & 36.3 & 30.5 & 0.54 & 1000 & 0.86 \\
\hline K22 & 0.58 & 0.78 & 37.0 & 31.0 & 0.55 & 1000 & 0.87 \\
\hline R23 & 0.58 & 0.77 & 38.1 & 31.5 & 0.54 & 1000 & 0.87 \\
\hline R24 & 0.59 & 0.76 & 36.6 & 31.3 & 0.57 & 1000 & 0.88 \\
\hline \multicolumn{8}{|l|}{ E25 } \\
\hline \multicolumn{8}{|l|}{ E26 } \\
\hline D27 & 0.54 & 0.73 & 39.0 & 33.4 & 0.52 & 920 & 0.88 \\
\hline E28 & 0.52 & 0.71 & 39.2 & 32.7 & 0.59 & 900 & 0.91 \\
\hline Q29 & 0.52 & 0.69 & 37.8 & 32.1 & 0.56 & 760 & 0.91 \\
\hline R32 & 0.52 & 0.70 & 39.3 & 32.4 & 0.56 & 820 & 0.91 \\
\hline \multicolumn{8}{|l|}{ K33 } \\
\hline E34 & 0.50 & 0.67 & 39.6 & 33.8 & 0.54 & 810 & 0.91 \\
\hline E35 & 0.51 & 0.71 & 39.1 & 32.7 & 0.58 & 830 & 0.91 \\
\hline E37 & 0.49 & 0.67 & 40.9 & 33.4 & 0.56 & 810 & 0.91 \\
\hline M40 & 0.50 & 0.68 & 38.9 & 32.6 & 0.53 & 710 & 0.91 \\
\hline L42 & 0.53 & 0.71 & 39.9 & 32.7 & 0.51 & 840 & 0.89 \\
\hline E43 & 0.56 & 0.72 & 37.7 & 32.1 & 0.51 & 870 & 0.88 \\
\hline M44 & 0.55 & 0.74 & 37.5 & 31.6 & 0.52 & 870 & 0.88 \\
\hline E45 & 0.57 & 0.76 & 37.1 & 31.9 & 0.50 & 890 & 0.87 \\
\hline A46 & 0.58 & 0.77 & 39.6 & 32.9 & 0.49 & 1000 & 0.85 \\
\hline K47 & 0.60 & 0.80 & 36.0 & 30.8 & 0.54 & 1000 & 0.86 \\
\hline R48 & 0.62 & 0.81 & 36.4 & 30.7 & 0.50 & 970 & 0.85 \\
\hline K49 & 0.65 & 0.85 & 35.1 & 28.3 & 0.48 & 930 & 0.84 \\
\hline Q50 & 0.65 & 0.86 & 35.4 & 29.9 & 0.49 & 1000 & 0.83 \\
\hline E51 & 0.66 & 0.85 & 35.4 & 29.5 & 0.48 & 1000 & 0.82 \\
\hline E52 & 0.69 & 0.89 & 33.4 & 28.4 & 0.47 & 1000 & 0.81 \\
\hline
\end{tabular}




$\begin{array}{llllllll}\text { E53 } & 0.70 & 0.89 & 33.9 & 28.5 & 0.46 & 1000 & 0.80 \\ \text { E45 } & 0.71 & 0.90 & 33.6 & 27.8 & 0.43 & 1000 & 0.79 \\ \text { K56 } & 0.80 & 1.00 & 30.4 & 25.4 & 0.40 & 1000 & 0.76 \\ \text { K57 } & 0.82 & 1.02 & 28.0 & 23.5 & 0.36 & 930 & 0.75 \\ \text { E59 } & 0.93 & 1.15 & 25.0 & 20.9 & 0.33 & 980 & 0.69 \\ \text { D60 } & 0.97 & 1.17 & 22.8 & 19.7 & 0.29 & 940 & 0.66 \\ \text { D61 } & 1.01 & 1.21 & 21.1 & 18.6 & 0.24 & 910 & 0.64 \\ \text { E62 } & 1.08 & 1.28 & 18.4 & 15.6 & 0.23 & 900 & 0.62 \\ \text { K63 } & 1.15 & 1.36 & 15.1 & 12.6 & 0.18 & 850 & 0.58\end{array}$

R64

I65

Q66

A67

E68

${ }^{\text {a }}$ Measured at $600 \mathrm{MHz}{ }^{1} \mathrm{H}$ frequency

${ }^{\mathrm{b}}$ Measured at $800 \mathrm{MHz}{ }^{1} \mathrm{H}$ frequency

Table S5. Longitudinal $\left(\mathrm{T}_{1}\right)$ and transverse $\left(\mathrm{T}_{1}\right){ }^{13} \mathrm{C}^{\alpha}$ relaxation of the E68W MT at $600 \mathrm{MHz}$ in $20 \mathrm{mM}$ sodium phosphate, $2 \mathrm{mM}$ EDTA, $\mathrm{pH}=6.3$ and $20{ }^{\circ} \mathrm{C}$. $\mathrm{MT}^{13} \mathrm{C}^{\alpha} \mathrm{T}_{1}$ values were measured using the Freeman-Hill inversion-recovery experiment using a non-selective, hyperbolic secant ${ }^{13} \mathrm{C}$ inversion pulse. Uncertainties in the relaxation times are ca $3 \%$.

$\begin{array}{lll}\text { Residue } & \mathrm{T}_{1}(\mathrm{~ms})^{\mathrm{a}} & \mathrm{T}_{1 \rho}(\mathrm{ms}) \\ \mathrm{E} / \mathrm{K} / \mathrm{R} / \mathrm{Q} & 698 & 26 \\ \mathrm{I} 13 & 713 & 35 \\ \mathrm{I} 65 & 438 & 79 \\ \mathrm{~A} 67 & 415 & 160\end{array}$


Table S6. Hydrogen exchange rates $\left(\mathrm{s}^{-1}\right)$ of the E68W MT domain in $20 \mathrm{mM}$ sodium phosphate, $2 \mathrm{mM}$ EDTA, $7 \% \mathrm{D}_{2} \mathrm{O}$, pH 7.8 at $20{ }^{\circ} \mathrm{C}$ and $30{ }^{\circ} \mathrm{C}$ and $\mathrm{pH} 8.9$ at $20{ }^{\circ} \mathrm{C}$

\begin{tabular}{|c|c|c|c|}
\hline Res & $20^{\circ} \mathrm{C} \mathrm{pH} 7.8$ & $30^{\circ} \mathrm{C} \mathrm{pH} 7.8$ & $20^{\circ} \mathrm{C} \mathrm{pH} 8.9$ \\
\hline E4 & 17 & & \\
\hline E5 & 7.5 & 20 & \\
\hline E6 & 6.7 & 20 & \\
\hline A7 & 3.0 & 10 & \\
\hline E8 & & 6.7 & \\
\hline R9 & 0.96 & 3.9 & 17 \\
\hline L10 & 0.59 & 2.0 & 6.6 \\
\hline R11 & 0.60 & 2.6 & 8.5 \\
\hline R12 & 0.75 & 4.0 & 14 \\
\hline 113 & 0.49 & & 5.6 \\
\hline Q14 & 0.59 & & 10 \\
\hline E15 & 0.57 & 2.5 & 9.6 \\
\hline E16 & 0.53 & & 7.9 \\
\hline M17 & 0.55 & 2.2 & 8.4 \\
\hline R18 & 0.78 & 3.5 & 16 \\
\hline E20 & 0.67 & & 10 \\
\hline K22 & 0.84 & 3.9 & 14 \\
\hline R23 & 0.84 & & 12 \\
\hline R24 & 1.1 & 4.0 & 20 \\
\hline E25 & 1.0 & 4.8 & 19 \\
\hline E26 & 0.85 & 3.4 & 13 \\
\hline D27 & 1.0 & 4.4 & 16 \\
\hline E28 & 0.82 & 3.3 & 13 \\
\hline Q29 & 0.77 & 2.9 & 12 \\
\hline R31 & 0.74 & 2.4 & 10 \\
\hline R32 & 0.77 & 3.5 & 11 \\
\hline K33 & 0.86 & 4.2 & 16 \\
\hline E34 & 0.63 & 2.6 & 9.4 \\
\hline E35 & 0.49 & 1.8 & 6.6 \\
\hline E36 & 0.36 & 1.1 & 3.3 \\
\hline E37 & 0.34 & 1.1 & 3.4 \\
\hline R39 & 0.56 & 2.3 & 7.4 \\
\hline M40 & 0.72 & 3.3 & 13 \\
\hline K41 & & 3.9 & \\
\hline L42 & 0.72 & 2.8 & 9.8 \\
\hline E43 & 0.90 & 3.7 & 14 \\
\hline M44 & 1.1 & 5.4 & 21 \\
\hline E45 & 1.2 & 5.2 & 21 \\
\hline A46 & 1.3 & 6.2 & 17 \\
\hline K47 & 1.4 & 6.5 & 20 \\
\hline
\end{tabular}




$\begin{array}{llll}\text { R48 } & 1.4 & 6.8 & 19 \\ \text { K49 } & 2.0 & 10 & 35 \\ \text { Q50 } & 2.3 & 12 & \\ \text { E51 } & 1.4 & 6.4 & 17 \\ \text { E52 } & 1.0 & 4.3 & 15 \\ \text { E53 } & 0.86 & 3.4 & 12 \\ \text { E54 } & 1.0 & 4.3 & 15 \\ \text { R55 } & & 7.2 & \\ \text { Q56 } & 2.3 & 11 & \\ \text { K57 } & 2.9 & 12 & \\ \text { R58 } & & 14 & \\ \text { E59 } & 6.2 & 21 & \\ \text { D60 } & 4.0 & & \\ \text { D61 } & 3.4 & 11 & \\ \text { E62 } & 3.4 & 10 & \\ \text { K63 } & & 16 & 3.9 \\ \text { R64 } & 11 & 30 & \\ \text { I65 } & 6.7 & 18 & \\ \text { Q66 } & 20 & & \\ \text { A67 } & 26 & 0.65 & \\ \text { W68 } & 0.22 & & \\ & & & \end{array}$


Table S7. ${ }^{1} D_{\mathrm{NH}} \mathrm{RDCs}$ measured at $20^{\circ} \mathrm{C}$ and $35^{\circ} \mathrm{C}, 900 \mathrm{MHz}$, for the DOTA-M8-Tm tagged I13C mutant of E68W MT in $20 \mathrm{mM}$ sodium phosphate, 2 mM EDTA, pH 6.3 and 7.0. Couplings ignore the negative sign of the ${ }^{15} \mathrm{~N}$ gyromagnetic ratio.

\begin{tabular}{|c|c|c|c|}
\hline Res & ${ }^{1} \mathrm{D}_{\mathrm{NH}}{ }^{20 \mathrm{C}, \mathrm{pH} 6.3}$ & ${ }^{1} \mathrm{D}_{\mathrm{NH}}{ }^{20 \mathrm{C}, \mathrm{pH} 7.0}$ & ${ }^{1} \mathrm{D}_{\mathrm{NH}}{ }^{35 \mathrm{C}, \mathrm{pH} 6.3}$ \\
\hline R24 & -26.0 & -26.0 & -20.9 \\
\hline E25 & -27.6 & -28.4 & -21.4 \\
\hline E26 & -14.9 & -14.8 & -9.4 \\
\hline D27 & -16.9 & -16.1 & -11.2 \\
\hline K28 & -30.6 & -29.3 & -20.4 \\
\hline Q29 & -21.2 & -22.3 & -14.6 \\
\hline R30 & -12.3 & -12.6 & -8.8 \\
\hline R31 & -20.8 & -20.2 & -15.1 \\
\hline R32 & -28.5 & -27.2 & -19.6 \\
\hline K33 & -15.1 & -16.3 & -10.6 \\
\hline E34 & -13.9 & -13.7 & -10.8 \\
\hline E35 & -24.2 & -24.1 & -17.0 \\
\hline E36 & -23.1 & -23.2 & -16.0 \\
\hline E37 & -13.1 & -13.6 & -9.2 \\
\hline R38 & -14.3 & -15.2 & \\
\hline R39 & -23.9 & -24.6 & -18.4 \\
\hline M40 & -18.4 & -18.8 & -12.3 \\
\hline K41 & -10.7 & -9.9 & -6.4 \\
\hline L42 & -18.1 & -18.0 & -12.6 \\
\hline E43 & -22.3 & -22.2 & -14.8 \\
\hline M44 & -12.4 & -11.8 & -7.2 \\
\hline E45 & -10.4 & -10.5 & -6.4 \\
\hline A46 & -20.2 & -20.3 & -13.0 \\
\hline K47 & -18.4 & -18.0 & -11.7 \\
\hline R48 & -9.0 & -9.5 & -5.3 \\
\hline K49 & -13.2 & -12.5 & -5.7 \\
\hline Q50 & -20.9 & -20.2 & -12.7 \\
\hline E51 & -13.1 & -13.2 & -7.6 \\
\hline E52 & -8.0 & -7.8 & -6.2 \\
\hline E53 & -15.5 & -15.5 & -9.0 \\
\hline E54 & -15.4 & -16.9 & -9.9 \\
\hline R55 & -8.5 & -8.1 & -4.1 \\
\hline K56 & -8.6 & -8.2 & -3.7 \\
\hline K57 & -16.6 & -16.5 & -9.4 \\
\hline R58 & -11.6 & -11.9 & -2.5 \\
\hline E59 & -5.4 & -4.9 & -1.9 \\
\hline D60 & -8.1 & -8.2 & -3.9 \\
\hline D61 & -12.6 & -12.2 & -5.1 \\
\hline E62 & -5.2 & -4.9 & -1.6 \\
\hline K63 & -2.4 & -2.4 & \\
\hline R64 & -7.9 & -7.8 & -3.0 \\
\hline 165 & -2.6 & -2.8 & -0.6 \\
\hline Q66 & 1.9 & 1.7 & 1.4 \\
\hline A67 & 0.3 & 0.3 & 0.3 \\
\hline W68 & & & -0.2 \\
\hline
\end{tabular}


Table S8. Alignment tensor (Saupe tensor) matrix elements from SVD fitting of experimental RDCs, upscaled for the effect of dynamics (main text) to the PDB coordinates of entry 6OBI, as obtained with the DC utility of NMRPipe.

\begin{tabular}{|c|c|c|c|c|c|c|c|c|c|c|}
\hline \multirow[b]{2}{*}{ Pf1 ${ }^{a}$} & \multicolumn{5}{|c|}{ Saupe Matrix $\left(\times 10^{4}\right)$} & \multicolumn{3}{|c|}{$\begin{array}{l}\mathrm{XYZ} \text { Tait-Bryan } \\
\text { angles }\left({ }^{\circ}\right)\end{array}$} & \multirow{2}{*}{\begin{tabular}{|l|}
$\begin{array}{l}\mathrm{Da} \\
\mathbf{( H z})\end{array}$ \\
-17.19
\end{tabular}} & \multirow{2}{*}{$\begin{array}{l}\mathbf{R h} \\
0.16\end{array}$} \\
\hline & -15.93 & -3.95 & 0.00 & 0.00 & 0.00 & 0.0 & 0.0 & 0.0 & & \\
\hline Pf1 ${ }^{b}$ & 8.43 & -0.46 & 1.42 & -1.07 & 3.22 & 13.4 & 4.0 & 42.4 & -9.98 & 0.24 \\
\hline $20^{c}$ & -13.40 & 18.17 & 2.36 & 0.35 & -8.88 & 29.3 & 2.5 & 82.1 & -19.86 & 0.50 \\
\hline $35^{d}$ & -9.79 & 4.55 & -0.31 & -0.31 & -6.51 & 23.2 & -1.2 & 95.2 & -13.59 & 0.41 \\
\hline
\end{tabular}

All tensors are obtained with the DC program of NMRPipe for residues 6 to 63 of the first conformer of entry 6OBI;

${ }^{a}$ Alignment tensor obtained from a simultaneous SVD fit of all $\left({ }^{1} D_{\mathrm{NH}},{ }^{2} D_{\mathrm{C}^{\prime} \mathrm{H},}{ }^{1} D_{\left.\mathrm{C}^{\prime} \mathrm{N}\right)} \mathrm{RDCs}(100\right.$ $\mathrm{mM} \mathrm{NaCl}$ );

${ }^{\mathrm{b}}$ Alignment tensor obtained from a SVD fit of ${ }^{1} \mathrm{D}_{\mathrm{NH}} \mathrm{RDCs}$ (no $\mathrm{NaCl}$ );

${ }^{\mathrm{c}}$ Alignment tensor obtained from a SVD fit of ${ }^{1} \mathrm{D}_{\mathrm{NH}} \mathrm{RDCs}$ collected at $900 \mathrm{MHz}$ for I13C MT, aligned by the DOTA-M8-Tm tag and $20^{\circ} \mathrm{C}$, after upscaling by $\exp [(+(i-13) / 49.75]$;

${ }^{\mathrm{d}}$ Alignment tensor obtained from a SVD fit of ${ }^{1} \mathrm{D} \mathrm{NH}$ RDCs collected at $900 \mathrm{MHz}$ for I13C MT, aligned by the DOTA-M8-Tm tag and $35^{\circ} \mathrm{C}$, after upscaling by $\exp [(+(i-13) / 36.63]$.

\section{References}

1. Halle, B.; Davidovic, M., Biomolecular hydration: From water dynamics to hydrodynamics. Proc. Natl. Acad. Sci. U. S. A. 2003, 100 (21), 12135-12140.

2. Garcia de la Torre, P.; Bloomfield, V. A., Hydrodynamic properties of complex, rigid biological macromolecules. Theory and applications. Quart. Rev. Biophys. 1981, 14, 81-139.

3. Tirado, M. M.; Garciadelatorre, J., Rotational-Dynamics of Rigid, Symmetric Top Macromolecules - Application to Circular-Cylinders. J. Chem. Phys. 1980, 73 (4), 1986-1993.

4. Wüthrich, K., NMR of Proteins and Nucleic Acids. John Wiley \& Sons: New York, 1986.

5. Schwieters, C. D.; Kuszewski, J. J.; Clore, G. M., Using Xplor-NIH for NMR molecular structure determination. Prog. Nucl. Magn. Reson. Spectrosc. 2006, 48 (1), 47-62.

6. Grishaev, A.; Bax, A., An empirical backbone-backbone hydrogen-bonding potential in proteins and its applications to NMR structure refinement and validation. J. Am. Chem. Soc. 2004, 126 (23), 7281-7292.

7. Ottiger, M.; Bax, A., Determination of relative N-H-N N-C ', C-alpha-C ', andC(alpha)$\mathrm{H}$-alpha effective bond lengths in a protein by NMR in a dilute liquid crystalline phase. $J$. Am. Chem.Soc. 1998, 120 (47), 12334-12341.

8. $\quad$ Landau, L. D.; Lifshitz, E. M., Statistical Physics. Third Edition ed.; Pergamon Press: 1980; Vol. 5. 
9. Roche, J.; Ying, J. F.; Shen, Y.; Torchia, D. A.; Bax, A., ARTSY-J: Convenient and precise measurement of (3) $\mathrm{J}(\mathrm{HNH}$ alpha) couplings in medium-size proteins from TROSYHSQC spectra. J. Magn. Reson. 2016, 268, 73-81.

10. Bohm, G.; Muhr, R.; Jaenicke, R., Quantitative analysis of protein far UV circular dichroism spectra by neural networks. Protein Eng. 1992, 5 (3), 191-195.

11. Freeman, R.; Hill, H. D. W., Fourier transform study of NMR spin-lattice relaxation by progressive saturation. J. Chem. Phys. 1971, 54 (8), 3367-3377.

12. Silver, M. S.; Joseph, R. I.; Hoult, D. I., Highly Selective Pi/2 and Pi-Pulse Generation. J. Magn. Reson. 1984, 59 (2), 347-351. 